\title{
Estrategia ecológica para reducir el impacto ambiental generado por el co2 emitido por aeronaves de la aviación policial
}

\author{
Ecological strategy to reduce the environmental impact of co2 emitted by of police \\ aviation aircrafts
}

Robinsson C. Torres ${ }^{*}$

Escuela de Aviación Policial

John E. Diaz. ${ }^{2}$

Escuela de Aviación Policial

Fecha recepción: 15 de enero de 2020

Fecha aceptación: 16 de mayo de 2020

(1) 2020 Universidad de Cordoba. Este es un artículo de acceso abierto distribuido bajo los términos de la licencia Creative Commons Attribution License, que permite el uso ilimitado, distribución y reproducció en cualquier medio, siempre que el autor original y la fuente se acreditan.

\section{RESUMEN}

El impacto ambiental que genera el gas contaminante (CO2) emitido por las aeronaves de instrucción de la Escuela de Aviación Policial, es un factor de afectación a la capa de ozono y conlleva consecuencias para la salud de los seres humanos, por lo que es necesario crear una estrategia ecologica con la cual se puedan mitigar

\section{ABSTRACT}

The environmental impact generated by the pollutant gas (CO2) emitted by instruction of Police Aviation School aircrafts, is a factor of affecting the ozone layer and carries consequences for the health of human beings, so it is necessary to create an ecological strategy that can mitigate the effects. With the investigation it was established that the

\footnotetext{
1 Técnico Profesional en Servicio de Policía / Professional Technician in Police Service. Estudiante de la Tecnología en Mantenimiento Aeronáutico / Student in Aeronutical Maintenance Technology. Escuela de Aviación Policial, Contiguo Aeropuerto José Celestino Mutis (San Sebastián de Mariquita, Tolima, Colombia). 3132929639 rc.torres00001@correo.policia.gov.co

2 Ingeniero Aeronáutico / Aeronautical Enginner. Coordinador de formación científica y tecnológica / Scientific and technological training coordinator. Escuela de Aviación Policial, (San Sebastián de Mariquita, Tolima, Colombia).jhon.diaz1030@correo.policia.gov.co.
} 
los efectos. Con la investigación se pudo establecer que los niveles de $\mathrm{CO} 2$ en la Escuela de Aviación Policial, ubicada en el municipio de San Sebastián de Mariquita, Tolima. Son más elevados de los que se recomienda en los estudios consultados por el grupo investigador, por tanto es fundamental la siembra de plantas purificadoras de aire en el área cartografiada. La investigación se encuentra estructurada en ocho (8) capítulos. El primero consiste el planteamiento y descripción de la situación problemática. El segundo comprende el análisis de los antecedentes literarios y teóricos que dan soporte a la investigación ya que aportan metodológica y epistemológicamente a la misma. Posteriormente se aborda el proceso metodológico. La investigación es de tipo cuasiexperimental y con un alcance de tipo descriptivo, ya que se diseñó un experimento pero no se pueden controlar ni manipular con rigor todas las variables. El capítulo cuarto analiza resultados con base en las características técnicas definidas en la metodología de investigación, el quinto nos habla de los indicadores de impacto en la implementación de la estrategia institucional, el sexto es el presupuesto, el séptimo contiene las conclusiones del proceso y el octavo es la bibliografía.

PALABRAS CLAVE: Aeronave, contaminación, dióxido de carbono, estrategia, reforestación.

\section{INTRODUCCIÓN}

El desarrollo de la actividad humana ha traído grandes problemas medioambientales. El transporte aéreo, a medida que avanza, se ha convertido en un contaminante importante, muestra de esto es que la gran empresa Boeing hace poco más de una década produzca un informe ambiental anual en el que explique mediante cifras sus esfuerzos por reducir y gestionar adecuadamente las emisiones contaminantes y demás sustancias nocivas que produce esta industria. El uso de las aeronaves en la Escuela de Aviación, ubicada en el municipio de Mariquita, Tolima, genera un efecto contaminante por los gases que emiten levels of $\mathrm{CO} 2$ in the Police Aviation School Aircrafts, located in city San Sebastian de Mariquita, Tolima. They are higher than that recommended in the studies consulted by the research group, the sowing of treatment plants for air in the mapped area is therefore fundamental. The research is structured into eight chapters (8). The first is the approach and description of the problem. The second includes the analysis of the literary and theoretical background that gives support to research since they provide methodological and epistemologically to the same. Subsequently the methodological process is addressed. Research is almost-experimental type, with a range of descriptive, since we designed an experiment but not you cannot control or manipulate rigorously all the variables. The fourth chapter examines results based on the technical specifications defined in methodology of research, fifth speaks about impact indicators in implementation of the institutional strategy and the sixth is the budget, the seventh It contains the conclusions in the process and the eighth is the bibliography.

KEYWORDS: Aircraft, pollution, carbon dioxide, strategy, reforestation. en sus procesos internos de combustión. El presente estudio hace un aporte social en cuanto a que contribuye al conocimiento para mejorar la calidad de vida de la comunidad, pues que su objetivo principal es dar cuenta de las consecuencias que la actividad dentro de esta zona tiene para ella, en especial para la comunidad que convive dentro de esta Escuela, además se propone una estrategia para purificar el aire contaminado por la emoción de $\mathrm{CO} 2$ durante la operación de aeronaves.

El cuidado del medio ambiente está por doquier en las políticas públicas internacionales (PNUMA, 2019), en ocasiones más como instrumentos de propaganda que como elementos propios de una nación con 
visión de futuro. Sin embargo, es indiscutible que a nivel mundial hay una tendencia por la protección del planeta, o por lo queda de él, como lo indica la Organización de las Naciones Unidas para la Agricultura y la Alimentación FAO (2015):

"Las medidas de protección han sido fundamentales en los procesos de recuperación ambiental a nivel mundial, las naciones han venido implementando algunas acciones para mitigar el daño que ellas mismas han causado. "En los decenios pasados se ha experimentado un incremento masivo de normas medioambientales, adoptadas en foros internacionales, como los acuerdos mundiales o regionales y las leyes promulgadas por la Comunidad Europea (CE), así como el consiguiente mayor impacto de tales fuentes en los sistemas jurídicos nacionales. Al mismo tiempo, se ha registrado una creciente tendencia hacia la transferencia de responsabilidades en el sector medioambiental desde los gobiernos centrales hacia las autoridades locales.".

La constitución de 1991 ampara el derecho a un ambiente sano (artículo 79); a un medio ambiente como patrimonio común (artículos 8 y 95) y a un desarrollo sostenible (artículo 80). La Policia Nacional de Colombia, en compromiso con la proteccion del medio ambiente, brinda su reconocimiento y empeño en salvaguardar los recursos, comprendiendo el ambiente como el medio en el cual desarrollamos nuestras actividades incluyendo los recursos naturales en general, y comprendiendo que durante la segunda cumbre de la tierra en 1992 se destaca que: "el deterioro del medio ambiente tiene implicaciones sociales, políticas y necesariamente económicas" (Policia Nacional de Colombia., 2017).

Las normas que controlan la contaminación atmosférica a nivel internacional, como la Conferencia de París (Comisión Europea, 2020), ha llevado a realizar investigaciones relacionadas con los métodos para controlar la emisión de gases contaminantes. Una de estas investigaciones es la titulada "Los sensores Químicos y su Utilidad en el Control de Gases
Contaminantes", realizada por Escalona, Manganiello, López y Vega (2012). En este texto se identificaron los siguientes gases considerados contaminantes: Monóxido de Carbono (CO), Dióxido de Nitrógeno (NO2), Ozono (O3) y Dióxido de Azufre (SO2), así como otros compuestos de impacto como Dióxido de carbono (CO2), los compuestos orgánicos volátiles (COV), hidrocarburos (HC), Óxido de Nitrógeno y de Azufre en general (NOX y SOX). Igualmente, Reinoso, Estrada y Sinche (2009), destacan que estos gases se caracterizan por conformar un grupo de elementos altamente contaminantes para la atmosfera del planeta y, por consiguiente, para el ambiente en general.

Por último y en aras de lograr justificar no únicamente el impacto de estos gases sino también la importancia investigativa alrededor de toda esta temática, se debe mencionar el trabajo de Cruz y Talero (2014). En esta investigación se logra evidenciar no sólo el efecto nocivo de estos gases en cuanto contribuyen al efecto invernadero, también las grandes dificultades que existen a la fecha para lograr medir el efecto de todos gases en el ambiente. Además, esta investigación logra dar cuenta de las consecuencias que las aeronaves tienen sobre la calidad del aire dentro de un aeropuerto.

Frente a la necesidad de controlar la emisión de estos gases contaminantes, se han desarrollado equipos de medición con sensores y sistemas de monitoreo como, por ejemplo, los químicos basados en transductores piezoeléctricos, electroquímicos y ópticos, los cuales permiten determinar la presencia de estos gases que afectan el medio ambiente e impactan la salud de quienes están expuestos a estos elementos químicos.

Las políticas de protección ambiental a nivel nacional han tenido un creciente desarrollo en los últimos años, las normas son extensas y los compromisos parecen inalcanzables. Se evidencia un aumento en la normatividad, el cual no se refleja en las acciones orientadas hacia el medio ambiente. Los proyectos de divulgación a nivel nacional han estado abanderados por el Ministerio del 
Medio Ambiente, donde se promueven acciones pedagógicas tendientes a al manejo adecuado de los recursos y al respeto por la naturaleza.

El programa pretende ampliar y desarrollar aún más la política nacional de educación ambiental proyectándola con mayor fuerza a nivel nacional y territorial para lograr que en el marco envolvente del Plan Nacional de Desarrollo se logre un crecimiento verde para la sociedad, en armonía con la naturaleza. Las Corporaciones Autónomas Regionales se unieron a la celebración y realizaron charlas educativas con las comunidades, talleres de liderazgo, foros, presentación de eventos culturales, expresiones artísticas, encuentros de jóvenes, entre otros, que pretenden crear conciencia sobre la necesidad de preservar los recursos naturales. El Ministerio de Ambiente logró incorporar la educación ambiental, sus instrumentos y estrategias en las diferentes políticas ambientales de Colombia estimulando la participación ciudadana en función de la construcción de una cultura ambiental desde las escuelas y comunidades apostando al desarrollo de procesos de cambio en los comportamientos de los individuos." (Ministerio del Medio Ambiente, 2.015).

Teniendo en cuenta que la generación y emisión de gases contaminantes especialmente dióxido de carbono (CO2) es uno de los principales causantes del efecto invernadero (lo que con el tiempo provocara el calentamiento global) (Ministerio de Ambiente, 2020), en este contexto surge la necesidad de reducir el impacto ambiental de las operaciones policiales realizadas con aeronaves, reconociendo la importancia de la conservación del medio ambiente y su entorno.

\section{MATERIALES Y METÓDOS}

Para determinar los niveles de contaminación se utilizó un medidor de gases Sperian PHD6, que es un detector de gases de tecnología de vanguardia. Es el detector de gases más configurable, con 5 puertos sensores, hasta 6 canales de detección y más de 19 opciones de sensor, que incluyen electroquímicos, LEL, PID e infrarrojos. Las operaciones cotidianas del detector se controlan totalmente mediante el botón de modo. Mediante una barra de estado en la pantalla con iconos fáciles de reconocer, el usuario puede consultar de inmediato todos los estados, desde el tiempo hasta la calibración. Los menús integrados y fáciles de navegar les permiten a los usuarios avanzados configurar el detector en el campo. (http://www.epibalear.es/archivos/c885ft_pdf .pdf).

El proyecto, desarrollado por Julián Giraldo \& Farid Durango (2019), tiene como caso de estudio la Escuela de Aviación Policial, dirección Contiguo Aeropuerto José Celestino Mutis, ubicada en el municipio de San Sebastián de Mariquita, en el departamento del Tolima, República de Colombia. (Figura 1)

Esta investigación actualmente no ha sido publicada ni ha sufrido modificaciones. El proyecto de investigación está divido en tres (04) fases, las cuales se observan en la tabla (Tabla 1).

Se realizó la identificación de tres zonas para realizar la medición de niveles de contaminación por $\mathrm{CO} 2$ tomándose:

Cafetería Institucional: Es un punto muy concurrido por toda la comunidad académica de la ESAVI, siendo un sitio de descanso y alimentación para los estudiantes. (Figura 2)

Coordenadas de ubicación.

N 5०12'23.7'"

W 74'53'10.5"

Plataforma: Es una de las zonas de obligatorio movimiento del personal, tanto de instrucción como de operación. (Figura 3)

Coordenadas de ubicación

N 512'22.9"'

W 745'0.7'"

Cerca de una aeronave: El personal de instrucción, se ve gran parte del día expuesto a los gases contaminantes debido a que su interacción es específicamente con las aeronaves. (Figura 4)

Coordenadas de ubicación

N 5'12'24.3'" 
W 74'53'06.9'"

Para determinar la cantidad de gases contaminantes producidos por las aeronaves. Se realizó la toma de 15 muestras en 5 días (tres muestras por día) a horarios de alto tráfico de operación aéreas, en los sitios mencionados anteriormente. Cabe resaltar que para el caso de la muestra en el entorno de una aeronave se realizó con el helicóptero Huey 11.

El instrumento de medición utilizado fue Sperian PHD6. Obteniendo los siguientes resultados de las tablas.

Medición en la Plataforma: El proceso de medición se realizó enfocando el Detector en dirección a la zona de la plataforma, puesto que es allí donde se producen la emisión de gases contaminantes.

Medición cerca de la Aeronave: La medición de la emisión de $\mathrm{CO} 2$ se realizó en el entorno de un helicóptero Huey 11 , al momento de encender el motor, para determinar el nivel de contaminación a la atmosfera.

\section{RESULTADOS Y DISCUSIÓN}

Objetivo específico 1. Determinar la cantidad promedio de (CO2) gas contaminante producido por las aeronaves de instrucción como Huey II, Bell 206 B3 y Cessna 172 en la Escuela de Aviación Policial.

Resultado esperado. Cuantificación de los niveles de (CO2) gas contaminante producido por las aeronaves de instrucción.

Objetivo específico 2. Establecer un jardín de plantas de reforestación, para filtrar el aire contaminado de $\mathrm{CO} 2$ emitido por determinadas aeronaves en la Escuela De Aviación Policial.

Resultado esperado. Construcción en sitio de un jardín de plantas purificadoras, reforestar con árboles captadores de $\mathrm{CO} 2$ zonas verdes para filtrar el aire contaminado de (CO2) gas emitido por determinadas aeronaves (Figura $5)$.

Objetivo específico 3. Materializar en lugares estratégicos de la Escuela de Aviación Policial, la instalación de plantas y árboles captadores de $\mathrm{CO} 2$ para purificar el aire, y así lograr a futuro la reducción del impacto ambiental generado por este gas contaminante.

Resultado esperado. Siembra de plantas y árboles captadores de $\mathrm{CO} 2$ en lugares estratégicos de la ESAVI.

\section{CONCLUSIONES}

Una vez ejecutado el proyecto de investigación se puede concluir que:

Se realizó un proyecto de investigación mediante la base de toma de muestras de niveles de contaminación el detector de gases Sperian PHD6. Un instrumento de medición especializado.

- Las muestras se tomaron con el instrumento debidamente calibrado según las normas internacionales.

- Se aplicaron los protocolos de medición del fabricante mediante la capacitación de la empresa contratada para el alquiler del dispositivo.

- Se determinaron los sitios de mayor flujo y aglomeración de personal como lo son; Cafetería, plataforma y un dato especializado en el entorno de una aeronave con el fin de identificar el nivel de $\mathrm{CO} 2$ emitido por un helicóptero HUEY II, al momento de encendido.

- Se determinaron 15 muestras, tres por día en 5 días de muestreos, estos se llevaron a cabo en tres sitios diferentes en tres horarios dispuestos de la siguiente manera: 9:00 am, 15:00 pm y 12:00 medio día.

- Se realizó de manera sistemática el plan de trabajo fijado por el grupo de investigación y se cumplieron todos los objetivos.

- Se postularon 2 estrategias de mitigación mediante la utilización de material vegetal filtrante de $\mathrm{CO} 2$. 
Se determinó realizar la siembra de especies forestales, arbustivas y forrajeras con el fin de mitigar el impacto por la exposición a $\mathrm{CO} 2$ del personal de instrucción.

Se seleccionaron dos líneas de acción de mitigación en concordancia con los sitios muestreados.

Se realizó la siembra de material vegetal en dos líneas diferentes. La primera mediante la instalación de un jardín con especies captadoras de $\mathrm{CO} 2$ en la zona de la plataforma, denominada el Bunker y la segunda mediante la siembra de árboles en tres zonas de la ESAVI.

cada una con unas especies filtrantes.

Se realizó la selección del material vegetal del jardín purificador mediante las especificaciones técnicas de adaptabilidad de las especies, es así como se determinó sembrar 50 individuos arbustivos (Croto) de condiciones fenotípicas robustas y resistentes a condiciones extremas y con beneficios filtrantes y paisajísticos, además de 200 plántulas de Maní forrajero con características de fácil propagación (trepadora) y condiciones resistencia a canciones de áreas degradadas.

Se realizó la obtención de beneficios secundarios con la siembra del jardín como lo es; mejoramiento de la calidad de vida, embellecimiento, control de erosión, restauración y zonas degradadas.

Se pudo determinar una proyección de captura de $\mathrm{CO} 2$ en un año de $1.500 \mathrm{~kg}$ de $\mathrm{CO} 2 /$ año.

En la segunda estrategia de mitigación se logró identificar 3 zonas con vocación de siembra de especies forestales especializadas en captura de $\mathrm{CO} 2$ esto debido a sus condiciones fenotípicas como; excesiva ramificación y hoja de gran tamaño.

Se seleccionaron plántulas en etapa Brinzal de especies; Ocobo, Roble, Guayacán, Nogal y Olivo Negro.

Se determinó un protocolo de espaciamiento de siembra de $6 \mathrm{~m} \mathrm{x}$ $6 \mathrm{~m}$ con el fin de obtener un óptimo desarrollo silvícola de los individuos arbóreos.

- Se determinó la aplicación de un arreglo agroforestal de Barrera Rompe viento. En una zona perimetral del ESAVI, con la que se buscó realizar una mitigación de las emisiones de $\mathrm{CO} 2$ a los barrios aledaños.

Se logró obtener una serie de beneficios secundarios como; embellecimiento para el caso de la zona 3 (Monumento de los Héroes Caídos en Combate), con la siembra de Ocobos los cuales florecen en su totalidad, ofreciendo una sensación de bienestar.

Se logró proyectar una barrera de purificación del aire contaminado en la zona de la Cafetería, con la siembra de especies forestales.

Se proyectó obtener un beneficio secundario en la zona de la cafetería mediante la disminución de la temperatura por efecto del sombrío de los árboles allí emplazados con el efecto del microclima.

- En total se proyectó la captura de $\mathrm{CO} 2$ en atapa adulta de reducción de contaminación del aire con la captura de $493 \mathrm{Kg}$ / año de CO2 con la siembra de las 3 zonas.

Se estableció un inventario forestal de las especies sembradas con el fin de realizar un seguimiento de mantenimiento para garantizar la continuidad del proyecto.

- Se proyectó la captura de $1.993 \mathrm{Kg}$

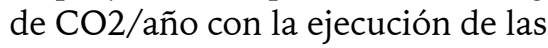


dos estrategias de mitigación; el Jardín Purificador y la siembra de especies forestales.

Se realizó la siembra de 326 árboles purificadores de aire.

Se entregó el manual de la estrategia y se socializo con la comunidad en general.

Con la investigación se pudo establecer que los niveles de $\mathrm{CO} 2$ en la Escuela de Formación Policial ESAVI, Ubicada en el Municipio de San Sebastián de Mariquita, Departamento del Tolima, son muy elevados presentando un alto grado de afectación a la salud de los Uniformados.

\section{REFERENCIAS}

[1]. Boeing, Resumen del informe medioambiental 2013.

[2]. Comisión Europea, "Acuerdo de París". Disponible en: https://ec.europa.eu/clima/policies/int ernational/negotiations/paris_es

[3]. Constitución Política de Colombia, 1991.

[4]. Durango, F, \& Giraldo, J. (2019). Estrategia ecológica para reducir el impacto ambiental generado por el (co2) gas contaminante que emiten las aeronaves de instrucción en la escuela de aviación policial (tesis de especialización). Escuela de aviación policial, Mariquita, Tolima.

[5]. Escalona, L., Manganiello, L., LópezFonseca, M., \& Vega, C. (2012). Los sensores químicos y su utilidad en el control de gases contaminantes. Revista Ingeniería UC, 19(1), 74-88.

[6]. Ficha técnica, Detector Biosystems
PHD6.

[7]. Fondo Nacional Ambiental. "Política y legislación ambiental". Disponible en: http://www.foronacionalambiental.org. co/nuestros-temas/politica-ambientalnacional/

[8]. Google Maps, (2020). San Sebastián de Mariquita.

[9]. Ministerio de Ambiente, (2020). "Gases efecto invernadero". Disponible en: http://www.minambiente.gov.co/index .php/component/content/article/462plantilla-cambio-climatico- 18

[10]. Ministerio del Medio Ambiente, 2015.

[11]. Organización de las Naciones Unidas para la Agricultura y la Alimentación F.A.O, 2015.

[12]. PNUMA, (2019). "Se registra gran aumento de leyes ambientales en los últimos 40 años, pero hace falta mejorar su aplicación, según nuevo reporte". Ciudad de Panamá.

[13]. Rodrigo, G. A., \& de Villa, A. B. R. (2012). El impacto ambiental del transporte aéreo y las medidas para mitigarlo. Trabajo de grado, Universidad Politécnica de Madrid, España). Recuperado de: http://oa. upm. es/20345/1/INVE_MEM_2012_13353 2. pdf.

[14]. Estrada Jiménez, J. C., \& Reinoso Chisaguano, D. J. (2009). Diseño e implementación de un prototipo de monitoreo remoto de contaminación ambiental utilizando tecnología GSM. (Artículo).

[15]. González Cruz, F. J., \& Talero Acosta, R. (2014). Estudio de impacto para el control y disminución de las emisiones de gases en los motores de los aviones, que contribuyen al efecto invernadero en el clima de Bogotá 